\title{
Frações de fósforo no solo após sucessivas aplicações de dejetos de suínos em plantio direto
}

\author{
Carlos Alberto Ceretta(1), Felipe Lorensini(1), Gustavo Brunetto(2), Eduardo Girotto(1), Luciano Colpo Gatiboni(3), \\ Cledimar Rogério Lourenzi( ${ }^{(1)}$, Tadeu Luis Tiecher ${ }^{(1)}$, Lessandro De Conti ${ }^{(1)}$, Gustavo Trentin ${ }^{(1)}$ e Alcione Miotto(1) \\ (1)Universidade Federal de Santa Maria, Centro de Ciências Rurais, Programa de Pós-Graduação em Ciência do Solo, Caixa Postal 221, \\ CEP 97105-900 Santa Maria, RS. E-mail: carlosceretta@gmail.com, felipe.lorensini@hotmail.com, eduardogirotto@hotmail.com, \\ crlourenzi@yahoo.com.br, tadeu.t@hotmail.com, lessandrodeconti@gmail.com, gustavotrentin@yahoo.com.br, alcionemiotto@gmail.com \\ (2)Universidade Federal de Santa Catarina, Centro de Ciências Agrárias, Departamento de Engenharia Rural, Rodovia Helmar \\ Gonzaga, no 1346, CEP 88034-000 Florianópolis, SC. E-mail: brunetto.gustavo@gmail.com (3)Universidade Estadual do Estado \\ de Santa Catarina, Departamento de Solos e Recursos Naturais, Avenida Luis de Camões, no 2090, CEP 88520-000 Lages, SC. \\ E-mail: gatiboni@cav.udesc.br
}

Resumo - O objetivo deste trabalho foi quantificar as frações de acumulação de fósforo em solo submetido a sucessivas aplicações de dejetos líquidos de suínos (DLS) em sistema de plantio direto. Em Santa Maria, RS, doses de 0, 20, 40 e $80 \mathrm{~m}^{3} \mathrm{ha}^{-1}$ de DLS foram distribuídas a lanço por sete anos agrícolas, antes do plantio de cada cultivo de inverno ou verão, em Argissolo Vermelho arenoso, totalizando 0, 584, 1.168 e $2.336 \mathrm{~kg} \mathrm{ha}^{-1}$ de P aplicado via dejetos. O solo foi coletado nas camadas $0-2,4-6,8-10,14-16$ e $20-25 \mathrm{~cm}$, e submetido ao fracionamento químico de P. A adição do DLS ao solo durante sete anos aumentou o teor de $\mathrm{P}$ até $25 \mathrm{~cm}$ de profundidade, principalmente nas frações inorgânicas extraídas por resina trocadora de ânions, $\mathrm{NaHCO}_{3}$ $0,5 \mathrm{~mol} \mathrm{~L}^{-1} \mathrm{e} \mathrm{NaOH} 0,1 \mathrm{~mol} \mathrm{~L}^{-1}$. As aplicações não aumentaram os teores de $\mathrm{P}$ orgânico extraído por $\mathrm{NaHCO}_{3}$ $0,5 \mathrm{~mol} \mathrm{~L}^{-1}$, mas sim as frações orgânicas extraídas por $\mathrm{NaOH} 0,5$ e $0,1 \mathrm{~mol} \mathrm{~L}^{-1}$. O DLS adicionado ao solo por longo período pouco afeta a partição de $\mathrm{P}$ em frações inorgânicas e orgânicas. As sucessivas aplicações de DLS aumentam o acúmulo de $\mathrm{P}$ em frações predominantemente lábeis no solo, o que representa um risco potencial para contaminação de águas superficiais e subsuperficiais.

Termos para indexação: esterco de suínos, fracionamento de fósforo, fósforo lábil, fósforo orgânico, risco de eutrofização, sistema de cultivo.

\section{Phosphorus fractions in soil after sucessive pig slurry applications in no-tillage system}

Abstract - The objective of this work was to quantify the accumulation of phosphorus fractions in the soil subjected to successive pig slurry applications in a no-tillage system. At Santa Maria, Rio Grande do Sul state, Brazil, 0, 20, 40 and $80 \mathrm{~m}^{3} \mathrm{ha}^{-1}$ doses of pig slurry were broadcasted for seven years on Argissolo Vermelho arenoso (Arenic Hapludult) before sowing each winter or summer crop, totalizing 0, 584, 1,168 and $2,336 \mathrm{~kg} \mathrm{ha}^{-1}$ of $\mathrm{P}$ from pig slurry. Soil samples were collected in $0-2,4-6,8-10,14-16$ and $20-25 \mathrm{~cm}$ layers and submitted to $P$ fractionation. Applications of pig slurry during seven years increased $\mathrm{P}$ contents to a depth of $25 \mathrm{~cm}$, especially in the inorganic fractions extractable by anion exchange resin, $\mathrm{NaHCO}_{3} 0.5 \mathrm{~mol} \mathrm{~L}^{-1}$ and $\mathrm{NaOH} 0.1 \mathrm{~mol} \mathrm{~L}^{-1}$. Pig slurry doses did not increase $\mathrm{P}$ content in organic fractions extracted by $\mathrm{NaHCO}_{3} 0.5$ mol L-1 , but increased the content of organic fractions extracted by $\mathrm{NaOH} 0.5 \mathrm{e} 0.1 \mathrm{~mol} \mathrm{~L}^{-1}$. Pig slurry applied to soil for large period has little effect on the P partition in inorganic and organic fractions. Successive pig slurry applications increase $\mathrm{P}$ contents in the soil predominantly in labile fractions, which can represent a potential risk for waterbeds contamination.

Index terms: pig manure, phosphorus fractionation, labile phosphorus, organic phosphorus, eutrophication risk, crop system.

\section{Introdução}

A suinocultura na região Sul do Brasil tem-se destacado como uma alternativa de renda nas pequenas propriedades com uso predominante de mão de obra familiar. O sistema de produçãoé intensivo, com grandes volumes de dejetos líquidos gerados anualmente. Esses dejetos podem ser utilizados na propriedade como fonte de nutrientes para culturas anuais ou pastagens. Porém, em razão da pequena extensão de terra das 
propriedades suinícolas, sucessivas aplicações de dejetos normalmente são realizadas na mesma área de cultivo, em muitos casos sobre solos rasos e declivosos. Com isso, a quantidade de $\mathrm{P}$ adicionada pode ultrapassar a capacidade de adsorção dos solos, e resultar no seu transporte para águas superficiais e subsuperficias, com alto risco de eutrofização (Adeli et al., 2003; Sharpley et al., 2004; Bergström \& Kirchmann, 2006; Gatiboni et al., 2008a).

Estudos sobre frações e acúmulo de $\mathrm{P}$ em solos tem sido realizados no Brasil com uso da técnica de fracionamento químico proposta por Hedley et al. (1982). Essa técnica usa extratores químicos aplicados sequencialmente em uma mesma amostra, para remoção progressiva das frações mais disponíveis até as mais estáveis de $\mathrm{P}$ inorgânico $(\mathrm{Pi})$ e orgânico (Po). A resina trocadora de ânions (RTA) inicia o fracionamento com a extração de frações lábeis de $\mathrm{P}$ inorgânico $\left(\mathrm{Pi}_{\mathrm{rta}}\right)$. $\mathrm{O}$ fracionamento continua com uso de $\mathrm{NaHCO}_{3}$ que também extrai frações lábeis de $\mathrm{P}$ inorgânico e orgânico $\left(\mathrm{Pi}_{\text {bic }}\right.$ e $\left.\mathrm{Po}_{\text {bic }}\right)$. Segundo Gatiboni et al. (2007), o P extraído por esses extratores pertence a frações que contribuem ativamente para o fornecimento do nutriente às plantas ou para sua transferência no ambiente, pelas águas de deflúvio ou de percolação.

Na sequência, o P é extraído pelo $\mathrm{NaOH} 0,1 \mathrm{~mol} \mathrm{~L}^{-1}$ ( $\mathrm{Pi}_{\text {hid }}$ e $\left.\mathrm{Po}_{\text {hid }}\right)$ e $\mathrm{NaOH}$ 0,5 mol L-1 $\left(\mathrm{Pi}_{\text {hid05 }}\right.$ e $\left.\mathrm{Po}_{\text {hid05 }}\right)$ que representam, em geral, o $\mathrm{P}$ inorgânico ligado aos óxidos e às argilas silicatadas, com energia de ligação intermediária, e o P orgânico de frações moderadamente lábeis (Cross \& Schlessinger, 1995). O HCl extrai o P inorgânico contido nos fosfatos de cálcio e fortemente adsorvido aos coloides. A digestão do solo com $\mathrm{H}_{2} \mathrm{SO}_{4}$ e $\mathrm{H}_{2} \mathrm{O}_{2}$ finaliza o processo de fracionamento com extração do $\mathrm{P}$ residual inorgânico + orgânico $\left(\mathrm{P}_{\text {residual }}\right)$ do solo, caracterizado por frações recalcitrantes de $\mathrm{P}$, que não contribuem para a nutrição das plantas a não ser em situações de extrema deficiência no solo (Gatiboni et al., 2005).

Com base no fracionamento químico de $\mathrm{P}$, tem-se observado que a adição de fertilizantes fosfatados ao solo resulta em acúmulo mais pronunciado de $\mathrm{P}$ nas frações inorgânicas, com diferentes graus de energia de ligação (Daroub et al., 2000; Santos et al., 2008; Pavinato et al., 2009). A adsorção do P ocorre primeiramente nos sítios mais ávidos pelo elemento e, posteriormente, o P remanescente é redistribuído em frações retidas com menor energia de ligação, de maior biodisponibilidade (Rheinheimer \& Anghinoni, 2001). Por sua vez, quando a fonte de fertilizante é de origem orgânica e de fácil decomposição, o acúmulo de $\mathrm{P}$ no solo ocorre primeiramente em frações orgânicas que somente migram para frações inorgânicas após mineralização. Contudo, caso os dejetos sejam compostos por material de difícil decomposição, a maior parte do $\mathrm{P}$ contido nele permanecerá no solo em frações orgânicas (Otabbong et al., 1997). Em solos submetidos à aplicação de dejetos, o acúmulo do $\mathrm{P}$ normalmente é mais pronunciado nas frações inorgânicas (Gatiboni et al., 2008a; Galvão \& Salcedo, 2009). Isso ocorre porque mais de $60 \%$ do $\mathrm{P}$ contido nos dejetos encontra-se em frações inorgânicas (Cassol et al., 2001). De acordo com Barnett (1994), em dejetos com $272 \mathrm{~g} \mathrm{~kg}^{-1}$ de matéria seca, a quantidade total de P é de, aproximadamente, $29,1 \mathrm{~g} \mathrm{~kg}^{-1}$, com $54,7 \%$ encontrado na forma inorgânica $(\mathrm{Pi})$ e o restante em frações orgânicas: $29,7 \%$ de hexafosfato de inositol (Po), 15,2\% de ácidos nucleicos (Po) e $0,4 \%$ de fosfolipídios (Po).

$\mathrm{O}$ conhecimento das formas de acumulação de $\mathrm{P}$ em solos que receberam sucessivas aplicações de dejetos de suínos é essencial para conhecer o real potencial de contaminação desse elemento. Isso poderá ser considerado na elaboração de estratégias adequadas para o uso de dejetos em sistemas de cultivo.

O objetivo deste trabalho foi quantificar as frações de acumulação de $\mathrm{P}$ em um solo submetido a sucessivas aplicações de dejetos líquidos de suínos em sistema plantio direto.

\section{Material e Métodos}

O experimento foi realizado na área experimental do Departamento de Engenharia Agrícola da Universidade Federal de Santa Maria, nas coordenadas geográficas $29^{\circ} 43^{\prime} \mathrm{S}$ e $53^{\circ} 42^{\prime} \mathrm{W}$, em Santa Maria, RS. O solo usado foi um Argissolo Vermelho distrófico arênico (Santos et al., 2006), textura superficial franco arenosa e relevo com declividade de $4 \%$.

O experimento foi implantado no ano 2000, em área cultivada há oito anos sob sistema de plantio direto. Em março de 2000, o solo na camada de 0-10 $\mathrm{cm}$ apresentava: $170 \mathrm{~g} \mathrm{~kg}^{-1}$ de argila composta de caulinita, e argilominerais do tipo interestratificado ilita-esmectita e caulinita-esmectita; $300 \mathrm{~g} \mathrm{~kg}^{-1} \mathrm{de}$ silte; $530 \mathrm{~g} \mathrm{~kg}^{-1}$ de areia; $\mathrm{pH}$ em água 4,7; matéria orgânica, $16 \mathrm{~g} \mathrm{~kg}^{-1}$; Al trocável, $0,8 \mathrm{cmol}_{\mathrm{c}} \mathrm{dm}^{-3}$; 
Ca trocável, 2,7 $\mathrm{cmol}_{\mathrm{c}} \mathrm{dm}^{-3} ; \mathrm{Mg}$ trocável, 1,1 $\mathrm{cmol}_{\mathrm{c}}$

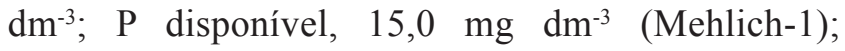
K trocável, 96,0 $\mathrm{mg} \mathrm{dm}^{-3}$; Cu e Zn (extraídos por $\mathrm{HCl}$ $\left.0,1 \mathrm{~mol} \mathrm{~L}^{-1}\right), 1,2$ e $1,6 \mathrm{mg} \mathrm{kg}^{-1}$, respectivamente.

Os tratamentos constituíram-se de 18 aplicações de dejetos líquidos de suínos, no período de março de 2000 até janeiro de 2008, nas doses de 0, 20, 40 e $80 \mathrm{~m}^{3} \mathrm{ha}^{-1}$. Os dejetos foram aplicadas antes da implantação de cada cultivo de inverno ou verão, no total de até três aplicações por ano. Após a colheita dos grãos ou floração das plantas de cobertura, as plantas foram dessecadas e os dejetos foram aplicados sobre os resíduos culturais. Os dejetos foram a única fonte de nutrientes fornecida às culturas. A caracterização dos dejetos usados em cada safra agrícola e cultura encontra-se na Tabela 1. Em janeiro de 2008, quando finalizado o experimento, a soma de fósforo fornecido via dejetos nas diversas aplicações correspondeu a 0 , $584,1.168$ e $2.336 \mathrm{~kg} \mathrm{ha}^{-1}$ de $\mathrm{P}$, para as doses de 0 , 20, 40 e $80 \mathrm{~m}^{3} \mathrm{ha}^{-1}$ de dejetos, respectivamente.

Ao longo do experimento, foram cultivadas as seguintes sucessões de plantios: aveia-preta (Avena strigosa S.), milho (Zea mays L.) e nabo-forrageiro (Raphanus sativus L.), nos anos agrícolas de 2000/2001 e 2001/2002 (Ceretta et al., 2005); aveia-preta, milheto(Pennisetum americanum L.) e feijão-preto (Phaseolus vulgaris L.), em 2002/2003; aveia-preta+ervilhaca (Vicia sativa L.) e milho, em 2003/2004 e 2004/2005; aveia-preta, feijão-preto, e crotalária (Crotalaria juncea L.) em 2005/2006; e aveia-preta e milho em 2006/2007. O delineamento experimental foi o de blocos ao acaso, com quatro

Tabela 1. Cultivos utilizados, percentagem de matéria seca (MS) dos dejetos líquidos de suínos e quantidade de fósforo aplicada ao longo dos anos, nos diferentes tratamentos.

\begin{tabular}{|c|c|c|c|c|c|c|c|c|c|}
\hline \multirow{2}{*}{$\begin{array}{l}\text { Características } \\
\text { de cada aplicação }\end{array}$} & \multicolumn{9}{|c|}{ Volume de dejetos $\left(\mathrm{m}^{3} \mathrm{ha}^{-1}\right)$ aplicado antes de cada cultivo } \\
\hline & 20 & 40 & 80 & 20 & 40 & 80 & 20 & 40 & 80 \\
\hline & \multicolumn{9}{|c|}{ Ano 2000/2001 } \\
\hline Cultivos & \multicolumn{3}{|c|}{ Aveia-preta } & \multicolumn{3}{|c|}{ Milho } & \multicolumn{3}{|c|}{ Nabo-forrageiro } \\
\hline MS dejetos (\%) & \multicolumn{3}{|c|}{1,9} & \multicolumn{3}{|c|}{9,9} & \multicolumn{3}{|c|}{0,8} \\
\hline \multirow[t]{2}{*}{$\mathrm{P}$ aplicado $\left(\mathrm{kg} \mathrm{ha}^{-1}\right)$} & 9,0 & 18,0 & 36,0 & 42,0 & 84,0 & 168,0 & 3,4 & 6,8 & 13,6 \\
\hline & \multicolumn{9}{|c|}{ Ano $2001 / 2002$} \\
\hline Cultivos & \multicolumn{3}{|c|}{ Aveia-preta } & \multicolumn{3}{|c|}{ Milho } & \multicolumn{3}{|c|}{ Nabo-forrageiro } \\
\hline MS dejetos $(\%)$ & \multicolumn{3}{|c|}{1,1} & \multicolumn{3}{|c|}{3,7} & \multicolumn{3}{|c|}{1,2} \\
\hline \multirow[t]{2}{*}{$\underline{\mathrm{P} \text { aplicado }\left(\mathrm{kg} \mathrm{ha}^{-1}\right)}$} & 3,3 & 6,6 & 13,2 & 12,0 & 24,0 & 48,0 & 3,1 & 6,2 & 12,4 \\
\hline & \multicolumn{9}{|c|}{ Ano $2002 / 2003$} \\
\hline Cultivos & \multicolumn{3}{|c|}{ Aveia-preta } & \multicolumn{3}{|c|}{ Milheto } & \multicolumn{3}{|c|}{ Feijão-preto } \\
\hline MS dejetos $(\%)$ & \multicolumn{3}{|c|}{0,5} & \multicolumn{3}{|c|}{6,7} & \multicolumn{3}{|c|}{4,8} \\
\hline \multirow[t]{2}{*}{$\underline{\mathrm{P} \text { aplicado }\left(\mathrm{kg} \mathrm{ha-}-^{1}\right)}$} & 2,0 & 4,0 & 8,0 & 15,9 & 31,8 & 63,6 & 28,4 & 56,8 & 113,6 \\
\hline & \multicolumn{9}{|c|}{ Ano $2003 / 2004$} \\
\hline Cultivos & \multicolumn{3}{|c|}{ Aveia-preta + ervilhaca } & \multicolumn{3}{|c|}{ Milho } & & - & \\
\hline MS dejetos (\%) & & 2,4 & & & 2,1 & & & - & \\
\hline $\mathrm{P}$ aplicado $\left(\mathrm{kg} \mathrm{ha}^{-1}\right)$ & 16,2 & 32,3 & 64,6 & 13,8 & 27,6 & 55,2 & & - & \\
\hline & & & & & o 2004/ & & & & \\
\hline Cultivos & & -preta $+c$ & laca & & Milho & & & - & \\
\hline MS dejetos (\%) & & 1,0 & & & 7,2 & & & - & \\
\hline P aplicado $\left(\mathrm{kg} \mathrm{ha}^{-1}\right)$ & 8,4 & 16,8 & 33,6 & 80,3 & 160,6 & 321,2 & & - & \\
\hline & & & & & o 2005 & & & & \\
\hline Cultivos & & Aveia-pr & & & Milhet & & & Feijão-p & \\
\hline MS dejetos (\%) & & 6,8 & & & 8,9 & & & 12,1 & \\
\hline P aplicado $\left(\mathrm{kg} \mathrm{ha}^{-1}\right)$ & 40,1 & 80,2 & 160,4 & 60,2 & 120,5 & 241,0 & 119,3 & 238,6 & 477,2 \\
\hline & & & & & o 2006/ & & & & \\
\hline Cultivos & & Aveia-pr & & & Milho & & & - & \\
\hline MS dejetos $(\%)$ & & 9,9 & & & 1,9 & & & - & \\
\hline $\mathrm{P}$ aplicado $\left(\mathrm{kg} \mathrm{ha}^{-1}\right)$ & 106,7 & 213,5 & 427,0 & 19,8 & 39,6 & 79,2 & & - & \\
\hline
\end{tabular}


repetições, e o tamanho de parcela de $4 \times 3 \mathrm{~m}$, com área útil de $8,75 \mathrm{~m}^{2}$.

Em janeiro de 2008, uma trincheira foi aberta no centro das parcelas de cada tratamento, para coleta de solo nas camadas $0-2,4-6,8-10,14-16$ e $20-25 \mathrm{~cm}$ de profundidade. Em seguida, o solo foi secado, moído passado em peneira com malha de $2 \mathrm{~mm}$. Na sequência, as amostras de solo foram submetidas ao fracionamento químico do $\mathrm{P}$, mediante extrações sucessivas, conforme Hedley et al. (1982), com as modificações propostas por Condron et al. (1985). Amostras de 0,5 g de solo seco foram submetidas à extração sequencial na seguinte ordem: resina trocadora de ânions em lâminas (AR 103 QDP 434) (fração $\mathrm{Pi}_{\mathrm{rta}}$ ); $\mathrm{NaHCO}_{3} 0,5$ mol L-1 (frações $\mathrm{Pi}_{\text {bic }}$ e $\mathrm{Po}_{\text {bic }}$ ); $\mathrm{NaOH}$ 0,1 mol L-1 (frações $\mathrm{Pi}_{\text {hid }}$ e $\mathrm{Po}_{\text {hid }}$ ); $\mathrm{HCl} 1,0 \mathrm{~mol} \mathrm{~L}^{-1}$ (fração $\mathrm{Pi}_{\mathrm{HCl}}$ ); e $\mathrm{NaOH}$ 0,5 mol L-1 (frações $\mathrm{Pi}_{\text {hid05 }}$ e $\mathrm{Po}_{\text {hid05 }}$ ). Após as extrações, o solo remanescente foi seco em estufa e submetido à digestão com $\mathrm{H}_{2} \mathrm{SO}_{4}+\mathrm{H}_{2} \mathrm{O}_{2}+\mathrm{MgCl}_{2}$ (fração $\mathrm{P}_{\text {residual }}$ ), conforme descrito em Gatiboni (2003).

O P inorgânico $(\mathrm{Pi})$ dos extratos alcalinos $\left(\mathrm{NaHCO}_{3}\right.$ e $\mathrm{NaOH}$ ) foi determinado pelo método proposto por Dick \& Tabatabai (1977). Nesses extratos alcalinos, o $P$ total foi determinado por digestão com persulfato de amônio + ácido sulfúrico, em autoclave, sendo o fósforo orgânico (Po) obtido pela diferença entre $\mathrm{P}$ total e o Pi. O P dos extratos ácidos foi determinado segundo Murphy \& Riley (1962). As frações de P determinadas no fracionamento de Hedley foram agrupadas em $\mathrm{P}$ geoquímico e biológico, de acordo com o proposto por Cross \& Schlesinger (1995), sendo o primeiro obtido pela soma das frações inorgânicas mais o $\mathrm{P}$ residual $\left(\mathrm{Pi}_{\text {rta }}\right.$ $\left.+\mathrm{Pi}_{\text {bic }}+\mathrm{Pi}_{\text {hid }}+\mathrm{Pi}_{\text {hid } 05}+\mathrm{Pi}_{\mathrm{HCl}}+\mathrm{P}_{\text {residual }}\right)$ e o segundo pela soma das frações orgânicas $\left(\mathrm{Po}_{\text {bic }}+\mathrm{Po}_{\text {hid }}+\mathrm{Po}_{\text {hido5 }}\right)$.

Os dados qualitativos, referentes ao teor de $\mathrm{P}$ em diferentes profundidades em uma mesma dose de dejetos líquidos de suínos, foram submetidos à análise de variância e, quando os efeitos foram significativos, compararam-se as médias pelo teste de Tukey a $5 \%$ de probabilidade. Os resultados quantitativos, referentes ao teor de $\mathrm{P}$ nas diferentes doses de dejetos líquidos de suínos, em uma mesma camada de solo, foram ajustados em equações de regressão, quando da significância dos efeitos apontados pela análise de variância.

\section{Resultados e Discussão}

As frações $\mathrm{Pi}_{\text {rta, }}, \mathrm{Pi}_{\text {bic }}$ e $\mathrm{Po}_{\text {bic }}$ diminuíram com o aumento da profundidade do solo, independentemente da dose de dejetos líquidos de suínos (DLS) utilizada (Tabela 2). Os teores de $\mathrm{P}$ nas frações $\mathrm{Pi}_{\text {rta }}$, e $\mathrm{Pi}_{\text {bic }}$ aumentaram linearmente com o aumento na dose de DLS aplicada. É interessante observar que, nessas frações, o coeficiente angular das regressões foi maior nas camadas superficiais e decresceu na medida em que a profundidade aumentou.

Pode-se constatar, portanto, maior efeito das doses de DLS nas camadas superficiais, o que era esperado em razão da forma de aplicação dos dejetos e da ausência de preparo do solo. $\mathrm{O}$ teor de $\mathrm{P}$ na fração $\mathrm{Po}_{\text {bic }}$, no entanto, não aumentou com as doses crescentes de DLS (Tabela 2). Isso pode ocorrer porque o acúmulo de P orgânico está condicionado ao aumento no teor de matéria orgânica, o que não ocorreu durante o período experimental. Além disso, a fração $\mathrm{Po}_{\text {bic }}$ está relacionada a compostos orgânicos de fácil decomposição, os quais sustentam e são regulados pela biomassa microbiana do solo, o que acarreta pequeno acúmulo nessa forma de $\mathrm{P}$ no solo, uma vez que ela pode ser rapidamente mineralizada (Chen et al., 2002; Gatiboni et al., 2007, 2008a, 2008b).

Entre os teores de $\mathrm{P}$ extraídos por hidróxido, as frações $\mathrm{Pi}_{\text {hid }}$ e $\mathrm{Po}_{\text {hid }}$ foram as mais representativas do acúmulo de $\mathrm{P}$ em razão da aplicação das doses de DLS, tendo ocorrido modificações menos expressivas nas frações $\mathrm{Pi}_{\text {hid05 }} \mathrm{e} \mathrm{Po}_{\text {hid05 }}$ (Tabela 3 ). Convém ressaltar que, com exceção dos teores na fração $\mathrm{P}_{\mathrm{o}_{\text {hid }}}$, na camada de 20-25 cm, o P nessas frações aumentou de forma linear com o aumento da dose de dejetos adicionada. Isso pode indicar que os coloides inorgânicos desse solo, em que predominam argilominerais como caulinita na fração argila, estão parcialmente saturados ou saturados com $\mathrm{P}$, uma vez que, em outros tipos de solos, especialmente naqueles que apresentaram alta quantidade de óxido de ferro, maiores quantidades de $\mathrm{P}$ mediamente lábil são encontradas na fração $\mathrm{Pi}_{\text {hid05, }}$ em razão do alto poder sortivo do solo (Gatiboni, 2003; Gatiboni et al., 2008a). As frações orgânicas medianamente lábeis $\left(\mathrm{Po}_{\text {hid }} \mathrm{e}\right.$ $\mathrm{Po}_{\text {hid05) }}$ ) mostram que há maior teor de $\mathrm{P}$ orgânico nas camadas mais superficiais do solo, provavelmente em razão do maior teor de matéria orgânica nessa camada (Gatiboni at al., 2008a). Diferentemente do observado na fração de Po mais lábil ( $\left.\mathrm{Po}_{\text {bic }}\right)$, a aplicação de dejetos aumentou de forma linear o Po nas frações $\mathrm{Po}_{\text {hid }}$, nas quatro primeiras camadas do solo, e $\mathrm{Po}_{\text {hido5, }}$, nas duas primeiras. 
Os teores de $\mathrm{P}$ extraídos por $\mathrm{HCl}$ 1,0 mol L-1 $\left(\mathrm{Pi}_{\mathrm{HCL}}\right)$ no solo sem adição de dejetos foram baixos (Tabela 4). Isso se explica porque o extrator $\mathrm{HCl}$ extrai preferencialmente frações de $\mathrm{P}$ de fosfatos de cálcio (Cross \& Schlessinger, 1995) e, como esse solo não possui abundância de minerais apatíticos, os teores naturais nessa forma são baixos. Por sua vez, com a adição de dejetos houve aumento nos teores de $\mathrm{P}$ extraído por esse extrator, também observado por Gatiboni et al. (2008a). Isso pode ser explicado pela composição dos dejetos, pois, de acordo com Cassol et al. (2001), mais de $60 \%$ do P contido nos DLS é extraído com $\mathrm{HCl} 1,0 \mathrm{~mol} \mathrm{~L}^{-1}$, por estar em frações inorgânicas ligadas ao cálcio.

Observa-se que o aumento em $\mathrm{Pi}_{\mathrm{HCl}}$, em razão da aplicação das doses de DLS, apresentou coeficiente angular relativamente pequeno (Tabela 4), quando comparado com o P extraído pelos demais extratores. Como o solo utilizado neste trabalho apresenta baixo teor de cálcio, o aumento dos teores de $\mathrm{Pi}_{\mathrm{HCl}}$ está muito associado ao acúmulo físico de dejetos na camada superficial do solo. Semelhantemente ao observado para os demais extratores, os teores de $\mathrm{Pi}_{\mathrm{HCl}}$ diminuem com a profundidade.

O P residual $\left(\mathrm{P}_{\text {residual }}\right)$, que é uma forma recalcitrante de $\mathrm{P}$ de difícil acesso pelos extratores químicos seletivos no solo, não foi afetado pela adição de dejetos de suínos (Tabela 4). Portanto, o P adicionado com dejetos de suínos acumula-se no solo em frações de menor recalcitrância. Gatiboni et al. (2008a) observaram que o $\mathrm{P}$ veiculado pela adição de até $40 \mathrm{~m}^{3}$ ha $^{-1}$ ano $^{-1}$ de dejetos de suínos apresenta apenas um pequeno acúmulo na fração $\mathrm{P}_{\text {residual }}$. Em solos arenosos e com menores proporções de óxidos de ferro e alumínio na fração argila, o fósforo adicionado tende a acumular-se preferencialmente em frações lábeis, com pouco ou nenhum efeito sobre a fração residual. Por sua vez, em solos com maior capacidade de adsorção de fosfatos, maiores proporções de $\mathrm{P}$ devem ser adsorvidas com alta energia, e somente extraídas com a digestão do solo (Cross \& Schlesinger, 1995).

A adição de dejetos aumentou especialmente os teores de $\mathrm{Pi}_{\text {rta, }}, \mathrm{Pi}_{\text {bic }}$ e $\mathrm{Pi}_{\text {hid }}$, que são frações de $\mathrm{P}$ lábeis (Figura 1). Aliado a esse fato, a ausência do

Tabela 2. Frações de fósforo extraídas pelos extratores resina trocadora de ânions (RTA) e $\mathrm{NaHCO}_{3} 0,5$ mol L-1, em diferentes camadas de solo submetido à aplicação de doses crescentes de dejeto líquido de suínos (DLS) ${ }^{(1)}$.

\begin{tabular}{|c|c|c|c|c|c|c|}
\hline \multirow{2}{*}{$\begin{array}{l}\text { Camada de solo } \\
(\mathrm{cm})\end{array}$} & \multicolumn{4}{|c|}{ Dose de DLS $\left(\mathrm{m}^{3} \mathrm{ha}^{-1}\right)$} & \multirow[t]{2}{*}{ Regressão } & \multirow[t]{2}{*}{$\mathrm{R}^{2}$} \\
\hline & 0 & 20 & 40 & 80 & & \\
\hline & \multicolumn{6}{|c|}{ Fósforo inorgânico extraído pela RTA (mg kg-1 $\left.\mathrm{Pi}_{\text {rta }}\right)$} \\
\hline $0-2$ & $68 \mathrm{a}$ & $312 \mathrm{a}$ & $581 \mathrm{a}$ & $879 \mathrm{a}$ & $y=106,000+10,114 x$ & $0,94 *$ \\
\hline $4-6$ & $24 b$ & $185 b$ & $491 b$ & $860 \mathrm{a}$ & $y=14,600+10,726 x$ & $0,99 *$ \\
\hline $8-10$ & $19 \mathrm{c}$ & $70 \mathrm{c}$ & $284 \mathrm{c}$ & $743 b$ & $y=-52,400+9,467 x$ & $0,96^{*}$ \\
\hline $14-16$ & $13 \mathrm{c}$ & $33 \mathrm{~cd}$ & $115 \mathrm{c}$ & $433 c$ & $y=-42,600+5,460 x$ & $0,77 *$ \\
\hline $20-25$ & $8 \mathrm{~d}$ & $17 \mathrm{~d}$ & $33 \mathrm{e}$ & $195 \mathrm{~d}$ & $y=-20,800+2,401 x$ & $0,86^{*}$ \\
\hline \multirow[t]{2}{*}{$\mathrm{CV}(\%)$} & 6,7 & 14,7 & 3,2 & 4,2 & - & - \\
\hline & \multicolumn{6}{|c|}{ Fósforo inorgânico extraído por $\mathrm{NaHCO}_{3}$ a $0,5 \mathrm{~mol} \mathrm{~L}^{-1}\left(\mathrm{mg} \mathrm{kg}^{-1}, \mathrm{Pi}_{\mathrm{bic}}\right)$} \\
\hline $0-2$ & $18 \mathrm{a}$ & $61 \mathrm{a}$ & $193 \mathrm{a}$ & $255 \mathrm{a}$ & $y=22,800+3,111 x$ & $0,92 *$ \\
\hline $4-6$ & $10 \mathrm{~b}$ & $46 b$ & $178 b$ & $219 b$ & $\mathrm{y}=16,330+2,767 x$ & $0,88^{*}$ \\
\hline $8-10$ & $10 \mathrm{~b}$ & $19 \mathrm{c}$ & $74 \mathrm{c}$ & $193 \mathrm{c}$ & $y=-10,330+2,410 x$ & $0,95^{*}$ \\
\hline $14-16$ & $9 b$ & $14 \mathrm{~d}$ & $28 \mathrm{~d}$ & $73 \mathrm{~d}$ & $y=1,600+0,838 x$ & $0,94 *$ \\
\hline $20-25$ & $5 \mathrm{~b}$ & $8 \mathrm{e}$ & $13 \mathrm{e}$ & $35 \mathrm{e}$ & $\mathrm{y}=1,800+0,384 \mathrm{x}$ & $0,97 *$ \\
\hline \multirow[t]{2}{*}{$\mathrm{CV}(\%)$} & 19,5 & 6,3 & 1,8 & 4,6 & - & - \\
\hline & \multicolumn{6}{|c|}{ Fósforo orgânico extraído por $\mathrm{NaHCO}_{3}$ a $0,5 \mathrm{~mol} \mathrm{~L}^{-1}\left(\mathrm{mg} \mathrm{kg}^{-1}, \mathrm{Po}_{\text {bic }}\right)$} \\
\hline $0-2$ & $31 \mathrm{a}$ & $63 \mathrm{a}$ & $24 \mathrm{a}$ & $46 a$ & ns & - \\
\hline $4-6$ & $22 b$ & $48 b$ & $27 \mathrm{a}$ & $33 b$ & ns & - \\
\hline $8-10$ & $18 \mathrm{~b}$ & $32 \mathrm{c}$ & $28 \mathrm{a}$ & $20 \mathrm{c}$ & ns & - \\
\hline $14-16$ & $18 \mathrm{~b}$ & $22 d$ & $26 a$ & $21 \mathrm{c}$ & ns & - \\
\hline $20-25$ & $16 b$ & $11 \mathrm{e}$ & $17 \mathrm{~b}$ & $17 \mathrm{c}$ & $\mathrm{ns}$ & - \\
\hline CV (\%) & 13,6 & 4,9 & 5,7 & 14,9 & - & - \\
\hline
\end{tabular}

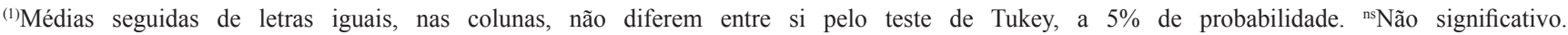
*Significativo a $5 \%$ de probabilidade. 
acúmulo de $\mathrm{P}$ em frações recalcitrantes reforça o seu potencial fertilizante, conforme relatado por Gatiboni et al. (2007), mas também aumenta seu potencial poluente. A adição de dejetos também aumentou os teores de $\mathrm{Po}_{\text {hid, }}$ que é mediamente lábil, mas em menor grau que as frações $\mathrm{Pi}_{\mathrm{rta}}, \mathrm{Pi}_{\text {bic }} \mathrm{e}$ $\mathrm{Pi}_{\text {hid. }}$ Esses dados se assemelham aos obtidos por Hountin et al. (2000), com a aplicação de 30, 60, 90 e $120 \mathrm{~m}^{3}$ ha $^{-1}$ ano $^{-1}$ de dejetos de suínos, durante 14 anos em um solo Gleissolo sob cultivo convencional. Para Gatiboni et al. (2008a), as frações de maior acúmulo foram $\mathrm{Pi}_{\text {rta }}, \mathrm{Pi}_{\text {bic }}$ e $\mathrm{Pi}_{\text {hid }}$ e, ainda, $\mathrm{Pi}_{\mathrm{HCl}}$, o que pode ser reflexo da composição dos dejetos utilizados, que proporcionou maior acúmulo de compostos de $\mathrm{P}$ ligados ao cálcio.

Tomando por base o ocorrido apenas na camada de 0 a $2 \mathrm{~cm}$ de profundidade, que se mostrou a mais afetada pela adição dos dejetos, os coeficientes angulares das equações de regressão mostram que, na fração $\mathrm{Pi}_{\text {rta }}$, houve um aumento de $10,1 \mathrm{mg} \mathrm{kg}^{-1}$ de $\mathrm{P}$ para cada $\mathrm{m}^{3}$ de dejetos adicionados; de $3,1 \mathrm{mg} \mathrm{kg} \mathrm{m}^{-1}$ para $\mathrm{Pi}_{\text {bic }} ; 3,5 \mathrm{mg} \mathrm{kg}^{-1} \mathrm{~m}^{-3}$ para $\mathrm{Pi}_{\text {hid }}$; $0,7 \mathrm{mg} \mathrm{kg}^{-1} \mathrm{~m}^{-3}$ para $\mathrm{Pi}_{\text {hid } 05} ; 1,0 \mathrm{mg} \mathrm{kg}^{-1} \mathrm{~m}^{-3}$ para $\mathrm{Pi}_{\mathrm{HCl}}$; $2,1 \mathrm{mg} \mathrm{kg}^{-1} \mathrm{~m}^{-3}$ para $\mathrm{Po}_{\text {hid }}$ e $1,0 \mathrm{mg} \mathrm{kg}^{-1} \mathrm{~m}^{-3}$ para $\mathrm{Po}_{\text {hid05 }}$. Fica evidente o maior acúmulo de $\mathrm{P}$ em frações inorgânicas no solo. Os resultados de $\mathrm{P}$ geoquímico, que é o somatório das frações inorgânicas de $P$,

Tabela 3. Frações de fósforo extraídas pelo extrator $\mathrm{NaOH}$ a 0,1 e a $0,5 \mathrm{~mol} \mathrm{~L}^{-1}$, em diferentes camadas de solo submetido à aplicação de doses crescentes de dejetos líquidos de suínos (DLS) ${ }^{(1)}$.

\begin{tabular}{|c|c|c|c|c|c|c|}
\hline \multirow{2}{*}{$\begin{array}{l}\text { Camada de solo } \\
(\mathrm{cm})\end{array}$} & \multicolumn{4}{|c|}{ Dose de DLS $\left(\mathrm{m}^{3} \mathrm{ha}^{-1}\right)$} & \multirow[t]{2}{*}{ Regressão } & \multirow[t]{2}{*}{$\mathrm{R}^{2}$} \\
\hline & 0 & 20 & 40 & 80 & & \\
\hline & \multicolumn{6}{|c|}{ Fósforo inorgânico extraído por $\mathrm{NaOH} 0,1 \mathrm{~mol} \mathrm{~L}^{-1}\left(\mathrm{mg} \mathrm{kg}^{-1}, \mathrm{Pi}_{\text {hid }}\right)$} \\
\hline $0-2$ & $102 \mathrm{a}$ & $180 \mathrm{a}$ & $285 \mathrm{a}$ & $377 \mathrm{a}$ & $y=114,670+3,464 x$ & $0,97 *$ \\
\hline $4-6$ & $98 \mathrm{ab}$ & $154 b$ & $252 b$ & $353 b$ & $y=100,270+3,257 x$ & $0,98 *$ \\
\hline $8-10$ & $89 \mathrm{bc}$ & $114 \mathrm{c}$ & $217 \mathrm{c}$ & $267 \mathrm{c}$ & $\mathrm{y}=89,000+2,367 \mathrm{x}$ & $0,91 *$ \\
\hline $14-16$ & $84 \mathrm{c}$ & $99 \mathrm{c}$ & $149 \mathrm{c}$ & $181 d$ & $\mathrm{y}=84,000+1,271 \mathrm{x}$ & $0,94 *$ \\
\hline $20-25$ & $63 \mathrm{~d}$ & $77 \mathrm{~d}$ & $108 \mathrm{e}$ & $137 \mathrm{e}$ & $\mathrm{y}=62,800+0,961 x$ & $0,97 *$ \\
\hline \multirow[t]{2}{*}{ CV $(\%)$} & 4,0 & 5,1 & 4,7 & 2,6 & - & - \\
\hline & \multicolumn{6}{|c|}{ Fósforo orgânico extraído por $\mathrm{NaOH}$ 0,1 mol L-1 (mg kg-1, $\left.\mathrm{Po}_{\mathrm{hid}}\right)$} \\
\hline $0-2$ & $77 \mathrm{a}$ & $143 \mathrm{a}$ & $168 \mathrm{a}$ & $256 a$ & $y=85,868+2,147 x$ & $0,98 *$ \\
\hline $4-6$ & $77 \mathrm{a}$ & $135 \mathrm{a}$ & $166 \mathrm{a}$ & $163 b$ & $y=100,930+0,988 x$ & $0,66^{*}$ \\
\hline $8-10$ & $60 \mathrm{~b}$ & $86 b$ & $59 b$ & $167 b$ & $y=48,800+1,263 x$ & $0,72 *$ \\
\hline $14-16$ & $48 \mathrm{c}$ & $63 \mathrm{bc}$ & $37 \mathrm{bc}$ & $77 \mathrm{c}$ & $y=45,932+0,290 x$ & $0,32 *$ \\
\hline $20-25$ & $29 d$ & $26 \mathrm{c}$ & $25 \mathrm{c}$ & $45 \mathrm{c}$ & ns & - \\
\hline \multirow[t]{2}{*}{ CV $(\%)$} & 7,1 & 18,5 & 12,9 & 13,2 & - & - \\
\hline & \multicolumn{6}{|c|}{ Fósforo inorgânico extraído por $\mathrm{NaOH} 0,5 \mathrm{~mol} \mathrm{~L}^{-1}\left(\mathrm{mg} \mathrm{kg}^{-1}, \mathrm{Pi}_{\text {hid05 }}\right)$} \\
\hline $0-2$ & $54 \mathrm{a}$ & $71 \mathrm{a}$ & $106 \mathrm{a}$ & $112 \mathrm{a}$ & $y=59,667+0,748 x$ & $0,85^{*}$ \\
\hline $4-6$ & $52 \mathrm{ab}$ & $63 b$ & $60 \mathrm{~b}$ & $82 b$ & $y=52,067+0,348 x$ & $0,89 *$ \\
\hline $8-10$ & $46 \mathrm{bc}$ & $55 \mathrm{bc}$ & $39 \mathrm{c}$ & $52 \mathrm{c}$ & ns & - \\
\hline $14-16$ & $43 c$ & $52 \mathrm{c}$ & $33 \mathrm{~cd}$ & $43 c$ & ns & - \\
\hline $20-25$ & $35 d$ & $40 d$ & $22 d$ & $25 d$ & $\mathrm{~ns}$ & - \\
\hline \multirow[t]{2}{*}{$\mathrm{CV}(\%)$} & 6,4 & 5,0 & 8,5 & 6,1 & - & - \\
\hline & \multicolumn{6}{|c|}{ Fósforo orgânico extraído por $\mathrm{NaOH} 0,5 \mathrm{~mol} \mathrm{~L}^{-1}\left(\mathrm{mg} \mathrm{kg}^{-1}, \mathrm{Po}_{\mathrm{hid} 05}\right)$} \\
\hline $0-2$ & $10 \mathrm{ab}$ & $17 \mathrm{a}$ & $32 \mathrm{a}$ & $86 a$ & $y=2,133+0,977 x$ & $0,94 *$ \\
\hline $4-6$ & $5 b$ & $9 b c$ & $58 \mathrm{a}$ & $49 b$ & $y=8,400+0,627 x$ & $0,62 *$ \\
\hline $8-10$ & $5 b$ & $3 \mathrm{~cd}$ & $27 \mathrm{bc}$ & $66 \mathrm{ab}$ & $y=-3,533+0,825 x$ & $0,93 *$ \\
\hline $14-16$ & $6 b$ & $1 d$ & $15 \mathrm{bc}$ & $15 \mathrm{c}$ & ns & - \\
\hline $20-25$ & $12 \mathrm{a}$ & $15 \mathrm{ab}$ & $12 \mathrm{c}$ & $10 \mathrm{c}$ & ns & - \\
\hline $\mathrm{CV}(\%)$ & 32,8 & 26,4 & 22,9 & 17,4 & - & - \\
\hline
\end{tabular}

${ }^{(1)}$ Médias seguidas de letras iguais, nas colunas, não diferem entre si pelo teste de Tukey, a $5 \%$ de probabilidade. ${ }^{\text {ns }}$ Não significativo. $*$ Significativo a $5 \%$ de probabilidade. 
Tabela 4. Fósforo inorgânico e residual, extraídos respectivamente pelos extratores $\mathrm{HCl} 1,0 \mathrm{~mol} \mathrm{~L}-1\left(\mathrm{Pi}_{\mathrm{HCl}}\right)$ e $\mathrm{H}_{2} \mathrm{SO}_{4}+\mathrm{H}_{2} \mathrm{O}_{2}+\mathrm{MgCl}_{2}$ $\left(\mathrm{P}_{\text {residual }}\right)$, em diferentes camadas de solo submetido à aplicação de doses crescentes de dejetos líquidos de suínos (DLS) ${ }^{(1)}$.

\begin{tabular}{|c|c|c|c|c|c|c|}
\hline \multirow{2}{*}{$\begin{array}{l}\text { Camada de solo } \\
(\mathrm{cm})\end{array}$} & \multicolumn{4}{|c|}{ Dose de DLS $\left(\mathrm{m}^{3} \mathrm{ha}^{-1}\right)$} & \multirow[t]{2}{*}{ Regressão } & \multirow[t]{2}{*}{$\mathrm{R}^{2}$} \\
\hline & 0 & 20 & 40 & 80 & & \\
\hline & \multicolumn{6}{|c|}{$\mathrm{Pi}_{\mathrm{HCl}}\left(\mathrm{mg} \mathrm{kg}^{-1}\right)$} \\
\hline $0-2$ & $17 \mathrm{a}$ & $54 a$ & $105 \mathrm{a}$ & $102 \mathrm{a}$ & $y=32,666+1,052 x$ & $0,74 *$ \\
\hline $4-6$ & $12 \mathrm{ab}$ & $28 \mathrm{~b}$ & $46 b$ & $75 a$ & $y=12,400+0,793 x$ & $0,99 *$ \\
\hline $8-10$ & $9 b$ & $14 \mathrm{c}$ & $23 b c$ & $37 \mathrm{~b}$ & $\mathrm{y}=7,934+0,364 \mathrm{x}$ & $0,99 *$ \\
\hline $14-16$ & $10 \mathrm{ab}$ & $10 \mathrm{~cd}$ & $20 \mathrm{c}$ & $35 \mathrm{bc}$ & $y=6,933+0,338 x$ & $0,94 *$ \\
\hline $20-25$ & $4 \mathrm{~b}$ & $5 \mathrm{~d}$ & $8 \mathrm{c}$ & $8 \mathrm{c}$ & ns & - \\
\hline \multirow[t]{2}{*}{ CV (\%) } & 27,9 & 9,1 & 20,9 & 18,7 & - & - \\
\hline & \multicolumn{6}{|c|}{$\mathrm{P}_{\text {residual }}\left(\mathrm{mg} \mathrm{kg}^{-1}\right)$} \\
\hline $0-2$ & $248 a b$ & $294 a$ & $295 \mathrm{a}$ & $239 a b c$ & ns & - \\
\hline $4-6$ & $268 \mathrm{a}$ & $268 \mathrm{a}$ & $276 a$ & $285 \mathrm{a}$ & $\mathrm{ns}$ & - \\
\hline $8-10$ & $234 b$ & $279 a$ & $188 b$ & $264 a b$ & ns & - \\
\hline $14-16$ & $175 \mathrm{c}$ & $234 \mathrm{a}$ & $222 b$ & $197 \mathrm{bc}$ & ns & - \\
\hline $20-25$ & $158 \mathrm{c}$ & $288 \mathrm{a}$ & $215 b$ & $183 \mathrm{c}$ & ns & - \\
\hline CV (\%) & 5,4 & 9,6 & 5,4 & 10,3 & - & - \\
\hline
\end{tabular}

${ }^{(1)}$ Médias seguidas de letras iguais, nas colunas, não diferem entre si pelo teste de Tukey, a $5 \%$ de probabilidade. ns Não significativo. ${ }^{*}$ Significativo a $5 \%$ de probabilidade.

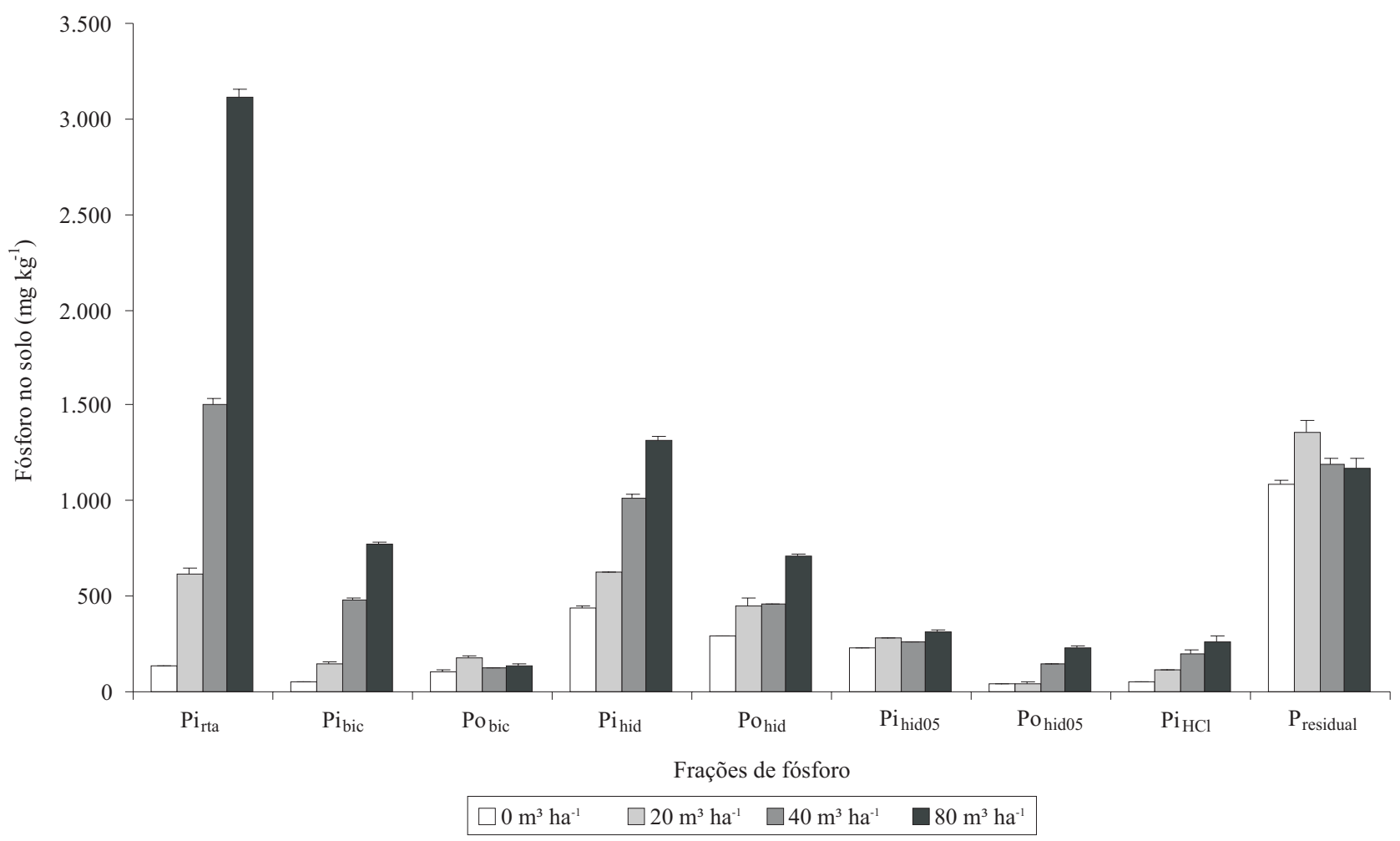

Figura 1. Somatório dos teores de fósforo das diferentes frações nas cinco camadas, em solo submetido à adição de doses crescentes de dejetos líquidos de suínos, ao longo de sete anos, em plantio direto. $\mathrm{Pi}_{\text {rta, }}$, fósforo inorgânico extraído pela resina; $\mathrm{Pi}_{\text {bic }}$, fósforo inorgânico extraído por $\mathrm{NaHCO}_{3}$ a $0,5 \mathrm{~mol} \mathrm{~L}^{-1} ; \mathrm{Po}_{\text {bic }}$, fósforo orgânico extraído por $\mathrm{NaHCO}_{3}$ a $0,5 \mathrm{~mol} \mathrm{~L}^{-1} ; \mathrm{Pi}_{\text {hid }}$, fósforo inorgânico extraído por $\mathrm{NaOH}$ a $0,1 \mathrm{~mol} \mathrm{~L}^{-1} ; \mathrm{Po}_{\text {hid }}$, fósforo orgânico extraído por $\mathrm{NaOH}$ a $0,1 \mathrm{~mol} \mathrm{~L}^{-1} ; \mathrm{Pi}_{\text {hid05}}$, fósforo inorgânico extraído por $\mathrm{NaOH}$ a $0,5 \mathrm{~mol} \mathrm{~L}^{-1} ; \mathrm{Po}_{\text {hid05 }}$, fósforo orgânico extraído por $\mathrm{NaOH}$ a

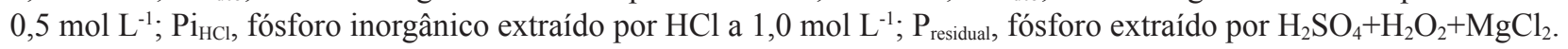


diminuíram com a profundidade do solo e aumentaram com a dose de dejetos aplicada (Tabela 5). Pode-se observar incrementos da ordem de 3,4 a $18,2 \mathrm{mg} \mathrm{kg}^{-1}$ de $\mathrm{P}$ por metro cúbico de DLS aplicado, dependendo da profundidade do solo. O P biológico, que é o somatório das frações orgânicas do fracionamento de Hedley, também aumentou com o aumento da dose de dejetos, exceto na maior profundidade. As magnitudes desse aumento variaram de 0,5 a $3,2 \mathrm{mg} \mathrm{kg}^{-1} \mathrm{~m}^{-3}$. Assim, as sucessivas aplicações de dejetos aumentaram a partição do $\mathrm{P}$ geoquímico de $81 \%$, onde não foram aplicados dejetos, para 87 e $83 \%$ do P total, nas doses de 40 e $80 \mathrm{~m}^{3}$ ha $^{-1}$ de dejetos, respectivamente.

$\mathrm{O}$ aumento do $\mathrm{P}$ total do solo foi significativo em todas as camadas, e acompanhou os teores de $\mathrm{P}$ geoquímico, já que o $\mathrm{P}$ total foi composto principalmente por frações inorgânicas (Tabela 5). Na camada 0-2 cm, incrementos de 568, 1.161 e $1.725 \mathrm{mg} \mathrm{kg}^{-1}$ de P para as doses de 20,40 e $80 \mathrm{~m}^{3} \mathrm{ha}^{-1}$ foram observados, respectivamente, em relação à testemunha sem aplicação. Isso representa um aumento de aproximadamente $275 \%$, nas aplicações sucessivas de $80 \mathrm{~m}^{3} \mathrm{ha}^{-1}$ de dejetos. Assim, mesmo que o P tenha alta afinidade pelos grupos de argilas e óxidos, quantidades tão expressivas devem ser adsorvidas com baixa energia de ligação, o que pode justificar a migração observada no $\mathrm{P}$ pelo perfil do solo, com consequente aumento nos seus teores totais até a camada de 20-25 $\mathrm{cm}$ de profundidade. Além disso, teores semelhantes nas camadas de 0-2 e 4-6 cm de profundidade indicam saturação dos grupos funcionais adsorventes de P nessas camadas. Galvão et al. (2008) constataram elevado potencial de perda de $\mathrm{P}$ por percolação profunda em razão da aplicação intensa de esterco bovino (15-20 $\mathrm{Mg} \mathrm{ha}^{-1}$ ao ano), durante períodos de até 40 anos, em solos arenosos do Nordeste, com migração de $\mathrm{P}$ a profundidades de até $60 \mathrm{~cm}$.

Após a adição de, aproximadamente, $2.336 \mathrm{~kg} \mathrm{ha}^{-1}$ de $\mathrm{P}$ via DLS, os teores de $\mathrm{P}$ na fração $\mathrm{Pi}_{\mathrm{rta}}$ na camada de $20-25 \mathrm{~cm}$ de profundidade ficaram acima de

Tabela 5. Fósforo geoquímico biológico e total ${ }^{(1)}$ em diferentes camadas de solo submetido à aplicação de doses crescentes de dejeto líquido de suínos (DLS) ${ }^{(2)}$.

\begin{tabular}{|c|c|c|c|c|c|c|}
\hline \multirow{2}{*}{$\begin{array}{l}\text { Camada de solo } \\
(\mathrm{cm})\end{array}$} & \multicolumn{4}{|c|}{ Dose de DLS $\left(\mathrm{m}^{3} \mathrm{ha}^{-1}\right)$} & \multirow[t]{2}{*}{ Regressão } & \multirow[t]{2}{*}{$\mathrm{R}^{2}$} \\
\hline & 0 & 20 & 40 & 80 & & \\
\hline & \multicolumn{6}{|c|}{ Fósforo geoquímico (mg kg-1) } \\
\hline $0-2$ & $507 a$ & $972 \mathrm{a}$ & $1.564 \mathrm{a}$ & $1.963 \mathrm{a}$ & $y=613,130+18,239 x$ & $0,94 *$ \\
\hline $4-6$ & $465 \mathrm{a}$ & $743 b$ & $1.302 \mathrm{~b}$ & $1.873 \mathrm{a}$ & $y=462,070+18,108 x$ & $0,98 *$ \\
\hline $8-10$ & $407 \mathrm{~b}$ & $551 \mathrm{c}$ & $820 \mathrm{c}$ & $1.554 \mathrm{~b}$ & $y=317,870+14,716 x$ & $0,97 *$ \\
\hline $14-16$ & $334 \mathrm{c}$ & $441 \mathrm{c}$ & $574 d$ & $964 c$ & $y=298,870+7,987 x$ & $0,98 *$ \\
\hline $20-25$ & $274 d$ & $436 \mathrm{c}$ & $399 \mathrm{e}$ & $582 d$ & $y=302,140+3,448 x$ & $0,86^{*}$ \\
\hline \multirow[t]{2}{*}{$\mathrm{CV}(\%)$} & 4,5 & 6,5 & 2,2 & 2,9 & - & - \\
\hline & \multicolumn{6}{|c|}{ Fósforo biológico (mg kg-1) } \\
\hline $0-2$ & $119 a$ & $223 a$ & $224 a$ & $389 a$ & $y=127,860+3,171 x$ & $0,94 *$ \\
\hline $4-6$ & $103 a$ & $193 a$ & $251 \mathrm{a}$ & $245 b$ & $y=140,070+1,658 x$ & $0,68 *$ \\
\hline $8-10$ & $83 b$ & $121 b$ & $115 b$ & $253 b$ & $\mathrm{y}=70,866+2,063 \mathrm{x}$ & $0,88^{*}$ \\
\hline $14-16$ & $72 \mathrm{bc}$ & $86 b c$ & $77 \mathrm{bc}$ & $112 \mathrm{c}$ & $y=70,532+0,468 x$ & $0,79 *$ \\
\hline $20-25$ & $58 \mathrm{c}$ & $52 \mathrm{c}$ & $54 \mathrm{c}$ & $72 \mathrm{c}$ & ns & - \\
\hline \multirow[t]{2}{*}{ CV (\%) } & 7,6 & 13,6 & 10,3 & 9,7 & - & - \\
\hline & \multicolumn{6}{|c|}{ Fósforo total $\left(\mathrm{mg} \mathrm{kg}^{-1}\right)$} \\
\hline $0-2$ & $627 \mathrm{a}$ & $1.195 \mathrm{a}$ & $1.788 \mathrm{a}$ & $2.352 \mathrm{a}$ & $y=741,140+21,410 x$ & $0,96^{*}$ \\
\hline $4-6$ & $568 \mathrm{a}$ & $935 b$ & $1.554 b$ & $2.119 b$ & $y=601,930+19,771 x$ & $0,97 *$ \\
\hline $8-10$ & $491 b$ & $672 c$ & $934 c$ & $1.806 \mathrm{c}$ & $y=388,930+16,771 x$ & $0,97 *$ \\
\hline $14-16$ & $456 c$ & $528 \mathrm{~cd}$ & $651 d$ & $1.077 \mathrm{~d}$ & $y=369,530+8,454 x$ & $0,98 *$ \\
\hline $20-25$ & $332 d$ & $489 d$ & $452 \mathrm{e}$ & $654 \mathrm{e}$ & $y=354,200+3,644 x$ & $0,87^{*}$ \\
\hline $\mathrm{CV}(\%)$ & 4,6 & 6,8 & 2,4 & 2,5 & - & - \\
\hline
\end{tabular}


$40 \mathrm{mg} \mathrm{kg}{ }^{-1}$ que, segundo a Comissão de Química e Fertilidade do Solo (CQFS-RS/SC) são interpretados como muito altos (Sociedade Brasileira de Ciência do Solo, 2004). Os teores de P extraído com esse extrator no tratamento com aplicação de $80 \mathrm{~m}^{3} \mathrm{ha}^{-1}$ de dejetos foram de $879,860,743,433$ e $195 \mathrm{mg} \mathrm{kg}^{-1}$, para as camadas de $0-2,4-6,8-10,14-16$ e $20-25 \mathrm{~cm}$ de profundidade, respectivamente (Tabela 2 ).

Assim, observa-se um grande incremento de $\mathrm{P}$ nas camadas superficiais e em profundidade no solo, o que aumenta o risco de perdas de fósforo via escoamento superficial e percolação, e potencializa processos de eutrofização de águas superficiais e subsuperficiais, como alertado por Basso et al. (2005) e Ceretta et al. (2005). Basso et al. (2005) observaram concentrações de $\mathrm{P}$ em soluções percoladas no perfil de, aproximadamente, $0,3 \mathrm{mg} \mathrm{L}^{-1}$, com uma única aplicação de $40 \mathrm{~m}^{3} \mathrm{ha}^{-1}$ de dejetos, em trabalho realizado na mesma área experimental. Ainda na mesma área experimental, Ceretta et al. (2005) observaram teores de até $14 \mathrm{mg} \mathrm{L}^{-1}$ de $\mathrm{P}$ disponível, em soluções escoadas na superfície do solo. A legislação brasileira estabelece o limite de $0,025 \mathrm{mg} \mathrm{L}^{-1}$ de P total na água, para que não haja riscos de eutrofização (Brasil, 1986).

\section{Conclusões}

1. As sucessivas aplicações de dejetos líquidos de suínos, na superfície de um Argissolo Vermelho arenoso, cultivado em sistema de plantio direto, aumenta o teor de fósforo até $25 \mathrm{~cm}$ de profundidade, principalmente nas frações inorgânicas extraídas por resina trocadora de ânions, $\mathrm{NaHCO}_{3}$ 0,5 mol L-1 e $\mathrm{NaOH}$ 0,1 $\mathrm{mol} \mathrm{L}^{-1}$.

2. A aplicação de dejetos líquidos de suínos não aumenta os teores de fósforo orgânico extraído por $\mathrm{NaHCO}_{3} 0,5$ mol L ${ }^{-1}$, porém aumenta as frações orgânicas extraídas por $\mathrm{NaOH} 0,1$ e $0,5 \mathrm{~mol} \mathrm{~L}^{-1}$.

3. O dejetos líquidos de suínos adicionado ao solo durante sete anos pouco afeta a partição de fósforo entre as frações inorgânicas e orgânicas.

4. As sucessivas aplicações de dejetos líquidos de suínos aumentam o acúmulo de fósforo em frações predominante lábeis no solo, e representa risco potencial de contaminação de águas superficiais e subsuperficiais.

\section{Referências}

ADELI, A.; VARCO, J.J.; ROWE, D.E. Swine effluent irrigation rate and timing effects on Bermudagrass growth, nitrogen and phosphorus utilization, and residual soil nitrogen. Journal of Environmental Quality, v.32, p.681-686, 2003.

BARNETT, G.M. Phosphorus forms in animal manure. Bioresource Technology, v.49, p.39-147, 1994.

BASSO, C.J.; CERETTA, C.A.; DURIGON, R.; POLETTO, N.; GIROTTO, E. Dejeto líquido de suínos: II - perdas de nitrogênio e fósforo por percolação no solo sob plantio direto. Ciência Rural, v.35, p.1305-1312, 2005.

BERGSTRÖM, L.; KIRCHMANN, H. Leaching and crop uptake of nitrogen and phosphorus from pig slurry as affected by different application rates. Journal of Environmental Quality, v.35, p.1803-1811, 2006.

BRASIL. Ministério do Desenvolvimento e Meio Ambiente. Resolução Conama $n^{\circ} 20$ de 10 jul. 1986. Diário Oficial da República Federativa do Brasil, 30 jul. 1986. 96p.

CASSOL, P.C.; GIANELlO, C.; COSTA, V.E.U. Frações de fósforo em estrumes e sua eficiência como adubo fosfatado. Revista Brasileira de Ciência do Solo, v.25, p.635-644, 2001.

CERETTA, C.A.; BASSO, C.J.; VIEIRA, F.C.B.; HERBES, M.G.; MOREIRA, I.C.L.; BERWANGER, A.L. Dejeto líquido de suínos: I - perdas de nitrogênio e fósforo na solução escoada na superfície do solo, sob plantio direto. Ciência Rural, v.35, p.1296-1304, 2005.

CHEN, C.R.; CONDRON, L.M.; DAVIS, M.R.; SHERLOCK, R.R. Phosphorus dynamics in the rhizosphere of perennial ryegrass (Lolium perenne L.) and radiata pine (Pinus radiata D. Don.). Soil Biology \& Biochemistry, v.34, p.487-499, 2002.

CONDRON, L.M.; GOH, K.M.; NEWMAN, R.H. Nature and distribution of soil phosphorus as revealed by a sequential extraction method followed by ${ }^{31} \mathrm{P}$ nuclear magnetic resonance analysis. Journal Soil Science, v.36, p.199-207, 1985.

CROSS, A.F.; SCHLESINGER, W.H. A literature review and evaluation of the Hedley fractionation: applications to the biogeochemical cycle of soil phosphorus in natural ecosystems. Geoderma, v.64, p.197-214, 1995.

DAROUB, S.H.; PIERCE, F.J.; ELLIS, B.G. Phosphorus fractions and fate of phosphorus-33 in soils under plowing and no-tillage. Soil Science Society of America Journal, v.64, p.170-176, 2000.

DICK, W.A.; TABATABAI, M.A. Determination of orthophosphate in aqueous solutions containing labile organic and inorganic phosphorus compounds. Journal of Environmental Quality, v.6, p.82-85, 1977.

GALVÃO, S.R. da S.; SALCEDO, I.H. Soil phosphorus fractions in Sandy soil amended with cattle manure for long periods. Revista Brasileira de Ciência do Solo, v.33, p.613-622, 2009.

GALVÃO, S.R. da S.; SALCEDO, I.H.; OLIVEIRA, F.F. de. Acumulação de nutrientes em solos arenosos adubados com esterco bovino. Pesquisa Agropecuária Brasileira, v.43, p.99-105, 2008.

GATIBONI, L.C. Disponibilidade de formas de fósforo do solo às plantas. 2003. 231p. Tese (Doutorado) Universidade Federal de Santa Maria, Santa Maria.

GATIBONI, L.C.; BRUNETTO, G.; KAMINSKI, J.; RHEINHEIMER, D. dos S.; CERETTA, C.A.; BASSO, C.J. 
Formas de fósforo no solo após sucessivas adições de dejeto líquido de suínos em pastagem natural. Revista Brasileira de Ciência do Solo, v.32, p.1753-1761, 2008a.

GATIBONI, L.C.; KAMINSKI, J.; RHEINHEIMER, D. dos S.; BRUNETTO, G. Fósforo da biomassa microbiana e atividade de fosfatases ácidas durante a diminuição do fósforo disponível no solo. Pesquisa Agropecuária Brasileira, v.43, p.1085-1091, 2008 b.

GATIBONI, L.C.; KAMINSKI, J.; RHEINHEIMER, D. dos S.; KAMINSKI, J.; FLORES, J.P.C. Biodisponibilidade de formas de fósforo acumuladas em solo sob sistema plantio direto. Revista Brasileira de Ciência do Solo, v.31, p.691-699, 2007.

GATIBONI, L.C.; RHEINHEIMER, D. dos S.; FLORES, A.F.C.; ANGHINONI, I.; KAMINSKI, J.; LIMA, M.A.S. de. Phosphorus forms and availability assessed by 31 P-NMR in successively cropped soil. Communications in Soil Science and Plant Analysis, v.36, p.2625-2640, 2005.

HEDLEY, M.J.; STEWART, J.W.B.; CHAUHAN, B.S. Changes in inorganic and organic soil phosphorus fractions induced by cultivation practices and by laboratory incubations. Soil Science Society of America Journal, v.46, p.970-976, 1982.

HOUNTIN, J.A.; KARAM, A.; COUILLARD, D.; CESCAS, M.P. Use of a fractionation procedure to assess the potential for $\mathrm{P}$ movement in a soil profile after 14 years of liquid pig manure fertilization. Agriculture, Ecosystems \& Environment, v.78, p.77-84, 2000.

MURPHY, J.; RILEY, J.P. A modified single solution method for the determination of phosphate in natural waters. Analytica Chimica Acta, v.26, p.31-36, 1962.
OTABBONG, E.; PERSSON, J.; IAKIMENKO, O.; SADOVNIKOVA, L. The ultuna long-term soil organic matter experiment. II. Phosphorus status and distribution in soils. Plant and Soil, v.195, p.17-93, 1997.

PAVINATO, S.P.; MERLIN, A.; ROSOLEM, C.A. Phosphorus fractions in Brazilian Cerrado soils as affected by tillage. Soil \& Tillage Research, v.105, p.149-155, 2009.

RHEINHEIMER, D. dos S.; ANGHINONI, I. Distribuição do fósforo inorgânico em sistemas de manejo de solo. Pesquisa Agropecuária Brasileira, v.36, p.151-160, 2001.

SANTOS, J.Z.L.; FURTINI NETO, A.E.; RESENDE, Á.V. de; CURI, N.; CARNEIRO, L.F.; COSTA, S.E.V.G. de A. Frações de fósforo em solo adubado com fosfatos em diferentes modos de aplicação e cultivado com milho. Revista Brasileira de Ciência do Solo, v.32, p.705-714, 2008.

SANTOS, H.G. dos; JACOMINE, P.K.T.; ANJOS, L.H.C. dos; OLIVEIRA, V.A. de; OLIVEIRA, J.B. de; COELHO, M.R.; LUMBRERAS, J.F.; CUNHA, T.J.F. (Ed.). Sistema brasileiro de classificação de solos. Rio de Janeiro: Embrapa Solos, 2006. 412p.

SHARPLEY, A.N.; MCDOWELL, R.W.; KLEINMAN, P.J.A. Amounts, forms, and solubility of phosphorus in soils receiving manure. Soil Science Society of America Journal, v.68, p.2048-2057, 2004.

SOCIEDADE BRASILEIRA DE CIÊNCIA DO SOLO. Núcleo Regional Sul. Comissão de Química e Fertilidade do Solo - RS/ SC. Manual de adubação e de calagem para os Estados do Rio Grande do Sul e Santa Catarina. 10.ed. Porto Alegre: SBCS, 2004. 400p.

Recebido em 16 de outubro de 2009 e aprovado em 23 de maio de 2010 